\title{
Novel Adaptive Blind-Equalizer-Order Selection Scheme for Multiple-Input Multiple-Output Channels
}

\author{
Kun Yan, Hsiao-Chun $\mathrm{Wu}$, Shih Yu Chang and Yiyan Wu \\ Electrical and Computer Engineering Department, Louisiana State University \\ Baton Rouge, Louisiana, 70803, U. S. A.
}

\begin{abstract}
This paper introduces a novel adaptive finiteimpulse-response (FIR) equalizer-order selection scheme for multiple-input multiple-output (MIMO) channels. The model-order selection for blind FIR equalizers is determined heuristically but hardly on any theoretical basis. However, this heuristical strategy would often lead to a suboptimal solution. Thus, we design a non-pilot-aided MIMO channellength estimation scheme and propose a new blind-equalizerorder determination scheme built on such an adaptive channel-length estimation method. Our simulations show that the proposed scheme greatly outperforms the conventional systems using the fixed heuristical model-order selection technique.
\end{abstract}

Index Terms-MIMO, channel length estimation, blind equalizer.

\section{INTRODUCTION}

Multi-input multi-output (MIMO) is one of the most promising techniques for achieving high data rate and reliability over the broadband wireless medium in recent years [1], [2], [3]. Using the spatial diversity provided by multiple paths, MIMO technique significantly improve channel capacity. However, with the increasing data rate of wireless applications, fading and multipath propagation might introduce intersymbol interference (ISI) in addition to additive white Gaussian noise (AWGN).

To deal with the ISI distortion, many equalizers, such as minimum mean-square error(MMSE) blind equalizer [4], equalizer based on Hidden Markov Models (HMM) [5], blind equalizer using high order statistics (HOS) [6], and Turbo equalizer [7], have attract researchers' eyes. Recently, equalizers for MIMO system have also been discussed in many literatures [8], [9]. Beside of the progress in practical area, some important theoretical properties for blind equalizer, such as, convergency of blind equalization algorithm with respect to optimization condition [10], and signal-to-interference ratio(SIR) with respect to equalizer's length are discussed [11], [12]. However, the scheme for choosing equalizer's order based on theoretical analysis have never been touched. In the existing literature, equalizers' length are mostly determined by heuristical experiments. Consequently, the equalization scheme might not be optimal for accuracy and computational complexity simultaneously.
To deal with this crucial problem, we would like to introduce a novel equalizer order selection scheme based on channel estimation. A non-pilot-aided channel-length estimation algorithm [13] based on the second-order statistics of the received signals from the MIMO transceiver is employed to estimate the longest channel impulse response (CIR). Then, the resulting MIMO channel-length information is used to estimate the channel impulse response. A novel scheme based on theoretical analysis of $L_{2}$ approximation error [12] is adopted to estimate length of blind equalizer consequently. With this novel equalizer choosing scheme, we can construct equalizer with optimal computational complexity and accuracy. Simulation results justify that our proposed order selection method greatly improve the system efficiency without sacrificing the equalizer's estimating accuracy.

The paper is organized as follows: System model is introduced in Section II, then the equalizer's order choosing scheme is briefly discussed in Section III. Simulation result will be presented in Section IV. Finally, conclusion is drawn in Section $\mathrm{V}$.

Notations: $\vec{A}$ denotes a vector and $\widetilde{A}$ denotes a matrix where $\widetilde{A}^{T}$ is its transpose. The set of all integers is denoted as $\mathcal{Z}$ and the statistical expectation operation is denoted as $\mathbf{E}\{\}$.

\section{MIMO-OFDM SySTEM MODEL}

We provide the transmission model for MIMO transmission system in this section. An $N_{t}$-transmitting $/ N_{r^{-}}$ receiving antenna configuration is considered. The signal at transmitting antenna $i$ is written as $\vec{s}_{i}[n]=\widetilde{F}_{N_{s}} \vec{a}_{i}[n]$, where $\vec{s}_{i}[n] \stackrel{\text { def }}{=}\left(s_{i}[n] s_{i}[n+1] \cdots s_{i}\left[n+N_{s}-1\right]\right)^{T}$, $\widetilde{F}_{N_{s}}$ is the $N_{s} \times N_{s}$ inverse discrete Fourier transform (IDFT) matrix, $N_{s}$ denotes the total number of subcarriers and $\vec{a}_{i}[n] \stackrel{\text { def }}{=}\left(a_{i}[n] a_{i}[n+1] \cdots a_{i}\left[n+N_{s}-1\right]\right)^{T}$ is the complex-valued symbol vector sent from antenna $i$ at time instant $n+N_{s}-1$. Assume that we collect $N+1$ received signal samples at each receiving antenna. The received signal $x_{j}[n]$ at receiving antenna $j$ is expressed as

$$
\begin{array}{r}
x_{j}[n]=\sum_{\substack{i=1 \\
N_{t}}}^{L_{j i}} h_{j i}[m] s_{i}[n-m]+\zeta_{j}[n], \\
n=0,1, \ldots, N, \quad j=1,2, \ldots, N_{r},
\end{array}
$$


where $h_{j i}[n]$ is a linear-time-invariant impulse response modeling the propagation path between the $i$ th transmitting and the $j$ th receiving antenna and $N \geq \max _{i, j}\left(L_{j i}\right)$ such that

$$
h_{j i}[n]=0, \text { for } n<0 \text { and } n>L_{j i} .
$$

We further define

$$
L_{j, \text { max }} \stackrel{\text { def }}{=} \max \left(L_{j 1}, L_{j 2}, \ldots, L_{j N_{t}}\right) .
$$

$\zeta_{j}[n]$ is the additive noise incurred at the $j$ th receiving antenna. We can write the matrix form for Eq. (1) as

$$
x_{j}[n]=\operatorname{trace}\left\{\widetilde{H}_{j} \widetilde{S}^{T}[n]\right\}+\zeta_{j}[n],
$$

where

$$
\begin{array}{r}
\widetilde{H}_{j} \stackrel{\text { def }}{=} 2 \sum_{k=1}^{K}\left\{\begin{array}{cccc}
h_{j 1}[0] & h_{j 1}[1] & \cdots & \\
\vdots & \vdots & \vdots & \\
h_{j \chi}[0] & h_{j \chi}[1] & \cdots & \\
\vdots & \vdots & \vdots & \\
h_{j N_{t}}[0] & h_{j N_{t}}[1] & \cdots & \\
h_{j 1}\left[L_{j 1}\right] & 0 & \cdots & 0 \\
\vdots & \vdots & \vdots & \vdots \\
\cdots & \cdots & \cdots & h_{j \chi}\left[L_{j, \max }\right] \\
\vdots & \vdots & \vdots & \vdots \\
h_{j N_{t}}\left[L_{j N_{t}}\right] & 0 & \cdots & 0
\end{array}\right\} .
\end{array}
$$

Note that $h_{j \chi}[n] \in\left\{h_{j i}[n] \mid L_{j i}=L_{j, \max }\right\}$.

$$
\begin{aligned}
& \widetilde{S}[n] \stackrel{\text { def }}{=} 2 \sum_{k=1}^{K}\left\{\begin{array}{cc}
s_{1}[n] & s_{1}[n-1] \\
\vdots & \vdots \\
s_{N_{t}}[n] & s_{N_{t}}[n-1]
\end{array}\right. \\
& \left.\begin{array}{cc}
\cdots & s_{1}\left[n-L_{j, \max }\right] \\
\vdots & \vdots \\
\cdots & s_{N_{t}}\left[n-L_{j, \max }\right]
\end{array}\right\}_{N_{t} \times\left(L_{j, \max }+1\right)}
\end{aligned}
$$

and trace $\{\quad\}$ denotes the trace operation of matrices. $\zeta_{j}[n]$ is assumed both spatially and temporally white, with zero mean and variance

$$
\mathbf{E}\left\{\left|\zeta_{j}[n]\right|^{2}\right\}=\sigma_{\zeta_{j}}^{2}
$$

Unlike the assumption of the identical delay and fading property in [14], [15], we assume that the delays $L_{j i}$ are not necessarily the same for all $i$.

\section{Novel Equalizer Order Selection Schema}

To determine the order for equalizer in MIMO system, we estimate the channel impulse response at first, then the equalizer's order is calculated according to a predetermined truncation error upper bound. Consequently this order selection scheme provide an effective way for finding the minimum equalizer length with given truncation error, a tradeoff between computational complexity and error caused by equalizer's truncation is obtained thereby.

\section{A. Non-Pilot-Aided Channel Estimation in MIMO system}

In this section, a blind channel impulse response estimation based on channel length estimation will be discussed. A novel channel length estimation algorithm has been studied in [13]. The Second order statistics for the received signal is used to estimated the channel length. We employ the estimated channel length to the iterative CIR estimation algorithm discussed in [16]. Without loss generality, we assume the MIMO channel to be linear-time-invariant(LTI) FIR filter with distinct length in the following.

\section{B. Novel Equalizer Order Selection Schema}

Based on the estimation in Section III-A, we introduce a novel scheme for choosing equalizer's order here. The theoretical analysis of the relationship between equalizer's order and equalizer's truncation error is presented in [12]. We briefly derive the truncation error for FIR equalizer based on the analysis in [12] here.

Given LTI channel impulse response as Eq. (2), we can easily factorize its z-transform $H_{j i}(z)$ as

$$
H_{j i}(z)=\frac{D(z)}{Q(z)},
$$

where $D(z)$ and $Q(z)$ are polynomial with variable $z^{-1}$. We can always factorize $D(z)$ as

$$
D(z)=\prod_{l_{1}=1}^{n_{>}}\left(z_{-1}-P_{l_{1}}\right)^{m_{l_{1}}} \prod_{l_{2}=1}^{n_{<}}\left(z_{-1}-p_{l_{2}}\right)^{m_{l_{2}}}
$$

where $\left|P_{l_{1}}\right|>1$ and $\left|p_{l_{2}}\right|<1, m_{l_{1}}, m_{l_{2}}$ denote the number of distinct roots $P_{l_{1}}$ and $p_{l_{2}}$. To simplify the derivation, we only consider the case when $m_{l_{1}}=m_{l_{2}}=$ 1, The other cases will be discussed in our following work. Considering these assumption, the z-transform for corresponding equalizer is

$$
\begin{aligned}
E(z)= & \frac{Q(z)}{\prod_{l_{1}=1}^{n_{>}}\left(z_{-1}-P_{l_{1}}\right) \prod_{l_{2}=1}^{n_{<}}\left(z_{-1}-p_{l_{2}}\right)} \\
= & \sum_{l_{1}=1}^{n_{>}} \frac{\rho_{l_{1}}}{\left(z^{-1}-P_{l_{1}}\right)}+\sum_{l_{2}=1}^{n_{<}} \frac{\eta_{l_{2}}}{\left(z^{-1}-P_{l_{2}}\right)} \\
& \quad+r_{0}+r_{1} z+r_{2} z^{2}+\ldots+r_{\Gamma} z^{\Gamma},
\end{aligned}
$$

where $\operatorname{deg}\left(r_{0}+r_{1} z+r_{2} z^{2}+\ldots+r_{\Gamma} z^{\Gamma}\right) \stackrel{\text { def }}{=} \Gamma$, and $\Gamma \stackrel{\text { def }}{=}$ $\operatorname{deg}(Q(z))-\operatorname{deg}(D(z))$ then the error caused by truncation is given by

$$
\begin{aligned}
\epsilon_{M, N}= & \sum_{k=M}^{M+\operatorname{deg}(D(z))-1}\left|\sum_{i_{1}=1}^{n>} F_{l_{1}, k}\right|^{2} \\
& +\sum_{k=N+\operatorname{deg}(D(z))-1}^{N}\left|\sum_{i_{2}=1}^{n<} f_{l_{2}, k}\right|^{2} .
\end{aligned}
$$


Note that

$$
\begin{aligned}
F_{l_{1}, k}= & \left.\frac{\rho_{l_{1}}\left(\frac{d^{\operatorname{deg}(D(z))-1}\left[\frac{D(z)}{\left(z^{-1}-P_{l_{1}}\right)}\right]}{d\left(z^{-1}\right)^{\operatorname{deg}(D(z))-1}}\right)}{(\operatorname{deg}(D(z))-1) !}\right|_{z^{-1}=0}\left(-\frac{1}{P_{l_{1}}^{M}}\right), \\
f_{l_{2}, k}= & \left.\frac{\eta_{l_{2}}\left(\frac{d^{1-\operatorname{deg}(D(z))}\left[\frac{D(z)}{\left(z^{-1}-P_{l_{2}}\right)}\right]}{d\left(z^{-1}\right)^{1-\operatorname{deg}(D(z))}}\right)}{(1-\operatorname{deg}(D(z))) !}\right|_{z^{-1}=0}\left(-p_{l_{2}}^{N}\right) .
\end{aligned}
$$

where $M \in \mathcal{Z}$ and $N \in \mathcal{Z}$ denote that $-N^{\text {th }}$ to $M^{\text {th }}$ indexed element in the correspondingly time domain series for $E(z)$ in $\mathrm{Eq}(10)$. We can define

$$
\begin{aligned}
& \Phi_{l_{1}, k}=\left.\frac{\rho_{l_{1}}\left(\frac{d^{\operatorname{dgg}(D(z))-1}\left[\frac{D(z)}{\left(z^{-1}-P_{l_{1}}\right)}\right]}{d\left(z^{-1}\right)^{\operatorname{deg}(D(z))-1}}\right)}{(\operatorname{deg}(D(z))-1) !}\right|_{z^{-1}=0},
\end{aligned}
$$

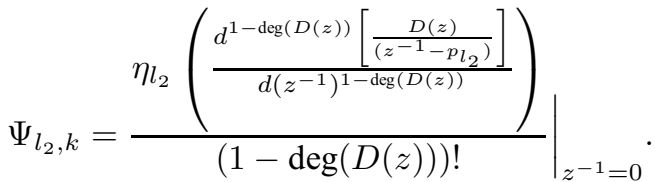

Clearly $\Phi_{l_{1}, k}$ and $\Psi_{l_{2}, k}$ are functions with respect to roots defined after Eq. (9). Then Eq. (11) can be simplified as

$$
\begin{aligned}
\epsilon_{M, N}= & \sum_{k=M}^{M+\operatorname{deg}(D(z))-1}\left|\sum_{i_{1}=1}^{n>}-\frac{\Phi_{l_{1}, k}}{P_{l_{1}}^{M}}\right|^{2} \\
& +\sum_{k=N+\operatorname{deg}(D(z))-1}^{N}\left|\sum_{i_{2}=1}^{n<}-\Psi_{l_{2}, k} p_{l_{1}}^{N}\right|^{2}
\end{aligned}
$$

From Eq. (14), we build the explicit truncation error function $\epsilon_{M, N}$ with respect to equalizer's order $M+N$, then, given an upper-bound for truncation error, the minimum equalizer length can be easily determined. For example, given an estimated $\mathrm{z}$-transform for a channel impulse response $h_{i j}(n)$ as follows

$$
H_{i j}(z)=\left(z^{-1}-0.5\right)\left(z^{-1}-2\right)\left(z^{-1}+1.5\right) .
$$

The truncation error can be derived as

$$
\begin{aligned}
& \epsilon_{M, N}=2.43 \times 0.25^{N} \\
& +\frac{9.98 \times 1.5^{2 M}+31 \times 2^{2 M}+36.2 \times 3^{M}}{3^{2 M}},
\end{aligned}
$$

Given an upper bound of truncation error $10^{-2}$, we can compute the minimum equalizer length as $M=11, N=$ 5 , Since $M \in \mathcal{Z}$ and $N \in \mathcal{Z}$, the result can be computed by discrete approximation.

We briefly state the workflow of our proposed order selection scheme as follows:
1. Estimate the maximum channel length according to the received signal;

2. Then Estimate the channel impulse response $h_{i j}(n)$ using the estimated channel length;

3. Decompose the corresponding Z-transform of $h_{i j}(n)$ and compute $\Phi_{l_{1}, k}$ and $\Psi_{l_{2}, k}$;

4. Given an upper bound of truncation error, calculate the minimum length of equalizer.

Since we assume the channel is time invariant in this paper, the equalizer order selection scheme can be carried out at the begin of equalization loop. On the other hand, when considering time variant channel, the selection loop might be perform occasionally to tracking the change of channel.

\section{Simulation}

A $2 \times 2$ MIMO-OFDM system is simulated $\left(N_{t}=N_{r}=\right.$ 2). Figure 1 illustrates the block diagram of the MIMOOFDM system. The channel estimation and order selection block is added in the receiver. In simulation we choose the channel parameters as follows: (1) the FFT/IFFT size is 256; (2) 16QAM is employed as the mapping constellation; (3) the channel coefficients are randomly generated while ITU pedestrian B model is used to characterize the power delay profile; (4) turbo code is used in order to achieve the maximum information transfer; (5) the maximum channel length of the 4 paths is 10 ; (6) the parameters in the channel-length estimator are set as: $\theta_{j}=0.01$ and $\Psi_{j}=0.0001, \forall j$. (7)channel estimation algorithm in [16] is employed in this simulation to estimate the channel. Minimum mean-square error (MMSE) equalizer is employed for the ultimate symbol detection according to [17]. The truncation error used in our simulation is chosen as 0.05 . Six iterations of turbo decoding scheme is adopted. We carry out 100 Monte Carlo trials to test our algorithm. We define a tradeoff for computational complexity and accuracy of the equalizer as

$$
\Upsilon=\frac{\varphi}{Q} \text {. }
$$

where $Q=\frac{1}{N_{r}} \sum_{\beta=1}^{N_{r}} W_{\beta}$ in MIMO system, $W_{\beta}$ is the length of each equalizer in MIMO receiver. $\varphi$ is given by

$$
\varphi=\mathbf{E}\left\{\frac{\max _{k}\left|w_{i j}(k) \otimes h_{i j}(k)\right|^{2}}{\sum_{k=0}^{Q+M}\left|w_{i j}(k) \otimes h_{i j}(k)\right|^{2}-\max _{k}\left|w_{i j}(k) \otimes h_{i j}(k)\right|^{2}}\right\},
$$

where the expectation in Eq. (18) is computed according all the transmitted channels. Figure 2 depicted the tradeoff defined here. Equalizer's length is chosen according to our novel order selection scheme. The tradeoff of the same system with fixed equalizer's order $2 L_{j, \max }-1$ is also 
shown in Figure 2. Clearly, the equalizer obtain similar SIR with shorter length when employ our novel order selection scheme.

\section{Conclusion}

In this paper, we propose a novel equalizer order selection scheme base on channel estimation and factorization. The novel scheme give us a theoretical analysis for determining the length of equalizer given a truncation error. Consequently the system could be optimal both in computational complexity and accuracy. A simulation for tradeoff in transmission system with our novel order selection algorithm is present to support our claim.

\section{REFERENCES}

[1] A. Goldsmith, S. Jafar, N. Jindal, and S. Vishwanath, "Capacity limits of mimo channels," IEEE Transactions on Selected Area in Communication, vol. 21, no. 5, pp. 684-702, June 2003.

[2] K. Yu, M. Bengtsson, B. Ottersten, D. McNamara, P. Karlsson, and M. Beach, "Modeling of wide-band mimo radio channels based on nlos indoor measurements," IEEE Transactions on Vehicular Technology, vol. 53, no. 3, pp. 655-665, May 2004.

[3] H. Zhang, M. T. Ivrlac, J. A. Nossek, and D. Yuan, "On multiuser mimo multi-stream transmission," IEEE Transactions on Vehicular Technology, 2009.

[4] J. K. Tugnait and B. Huang, "Blind channel estimation and equalization of multiple-input multiple-output channels," in Proceedings of IEEE International Conference on Personal Wireless Communication, vol. 17-19, Feb. 1999, pp. 231-235.

[5] H. Nguyen and B. C. Levy, "Blind and semi-blind equalization of cpm signals with the emv algorithm," IEEE Transactions on Signal Processing, vol. 51, no. 10, pp. 2650-2664, Oct. 2003.

[6] B. Mariere, Z.-Q. Luo, and T. N. Davidson, "Blind constant modulus equalization via convex optimization," IEEE Transactions on Signal Processing, vol. 51, no. 3, pp. 805-818, Mar. 2003.

[7] B. L. Yeap, H. W. Choong, and L. Hanzo, "Reduced complexity in-phase/quadrature-phase m-qam turbo equalization using iterative channel estimation," IEEE Transactions on Wireless Communication, vol. 2, no. 1, pp. 2-10, Jan. 2003.

[8] V. Kavitha and V. Sharma, "Comparison of training, blind and semi blind equalizers in mimo fading systems using capacity as measure," in Proceedings of IEEE International Conference on Acoustics, Speech, and Signal Processing, vol. 3, Mar. 2005, pp. 589-592.

[9] M. Shaodan and T.-S. Ng, "Semi-blind time-domain equalization for mimo-ofdm systems," IEEE Transactions on Vehicular Technology, vol. 57, no. 4, pp. 2219-2227, 2008.

[10] Y. Li and K. J. R. Lin, "On blind equalization of mimo channels," in Proceedings of IEEE International Conference on Communication, vol. 2, June 1996, pp. $1000-1004$.

[11] N. Al-Dhahir, "Fir channel-shortening equalizers for mimo isi channels," IEEE Transactions on Communications, vol. 49, no. 2, pp. 213-218, Feb. 2001.

[12] S. Y. Chang and H.-C. Wu, " $l_{2}$ approximation error evaluation for the inverse of mixing-phase systems and channel equalization applications," in Proceedings of IEEE International Conference on WCNC 2008, 2008, pp. 221-225.

[13] X. Wang, H.-C. Wu, S. Y. Chang, and Y. Y. Wu, "Efficient non-pilotaided channel length estimation for digital broadcasting receivers," IEEE Transactions on Broadcasting, vol. 55, no. 3, pp. 633-641, 2009.

[14] Z. J. Wang, Z. Han, and K. J. R. Liu, "A MIMO-OFDM channel estimation approach using time of arrivals," IEEE Transactions on Wireless Communications, vol. 4, no. 3, pp. 1207-1213, May 2005.

[15] H. Minn, N. Al-Dhahir, and Y. Li, "Optimal training signals for MIMO OFDM channel estimation in the presence of frequency offset and phase noise," IEEE Transactions on Communications, vol. 54, no. 10, pp. 1754-1759, October 2006.
[16] F. Moazzami and A. Cole-Rhoddes, "An adaptive blind equalizer with signal separation for a mimo system transmitting qam signals," in Proceedings of IEEE International Conference on Military Communication Conference 2008, Nov. 2008, pp. 1-5.

[17] A. F. Molisch, Wideband Wireless Digital Communications. Prentice Hall, 2000.
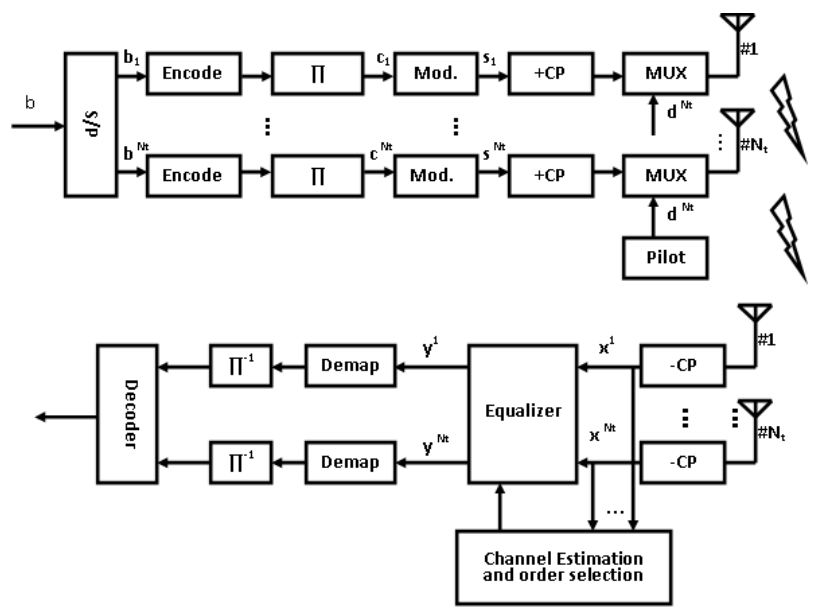

Fig. 1. Block diagram of the transmission system

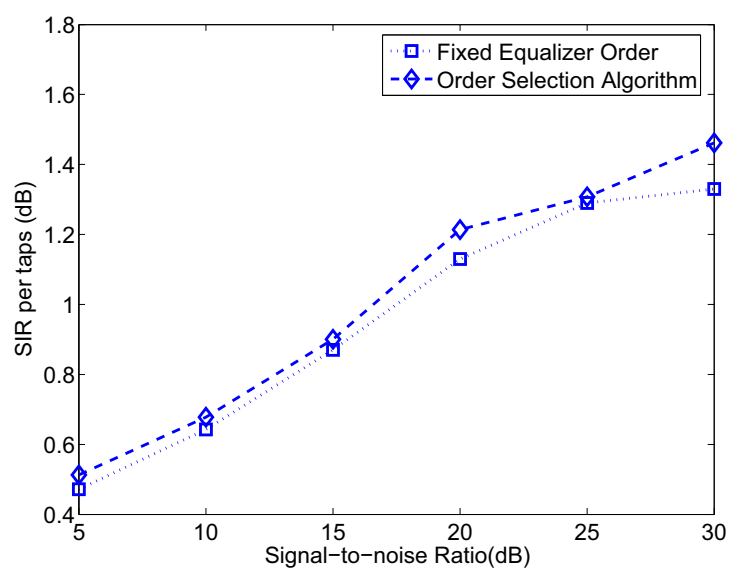

Fig. 2. Tradeoff comparison between our proposed order selection algorithm and the fixed equalizer order. The equalizer length is fixed as $2 L_{j, \max }-1$ in the conventional algorithm. The truncation error used in our order selection algorithm is chosen as 0.05 . 